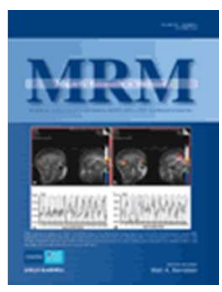

\title{
Perfusion MRI quantification in stroke: to deconvolve, or not to deconvolve
}

\begin{tabular}{|r|l|}
\hline Journal: & Magnetic Resonance in Medicine \\
\hline Manuscript ID: & Draft \\
\hline Datey - Manuscript type: & Full Paper \\
\hline Complete List of Authors: & $\begin{array}{l}\text { Meijs, Midas; Eindhoven University of Technology, } \\
\text { Christensen, Soren; Stanford University School of Medicine, } \\
\text { Lansberg, Maarten; NeurologNeurology and Neurological Sciences, } \\
\text { Neurology } \\
\text { Albers, Gregory; Stanford University School of Medicine, } \\
\text { Calamante, Fernando; The Florey Institute of Neuroscience and Mental } \\
\text { Health, }\end{array}$ \\
\hline Research Type: & $\begin{array}{l}\text { Perfusion < Flow and perfusion < Technique Development < Technical } \\
\text { Research }\end{array}$ \\
\hline Research Focus: & Brain < Neurological \\
\hline \multicolumn{2}{|l}{} \\
\hline
\end{tabular}


Title: Perfusion MRI quantification in stroke: to deconvolve, or not to deconvolve

Authors: Midas Meijs, ${ }^{1}$ Soren Christensen, ${ }^{2}$ Maarten G., Lansberg, ${ }^{2}$ Gregory W. Albers, ${ }^{2}$ Fernando Calamante $e^{3,4,5}$

${ }^{1}$ Eindhoven University of Technology, Eindhoven, The Netherlands.

${ }^{2}$ Stanford Stroke Center, Stanford University School of Medicine, Stanford, CA, USA.

${ }^{3}$ The Florey Institute of Neuroscience and Mental Health, Melbourne, Australia.

${ }^{4}$ The Florey Department of Neuroscience and Mental Health, University of Melbourne, Melbourne, Australia.

${ }^{5}$ Department of Medicine, Austin Health and Northern Health, University of Melbourne, Melbourne, Australia.

Corresponding author: Fernando Calamante, Florey Institute of Neuroscience and Mental Health, Melbourne Brain Centre, 245 Burgundy Street, Heidelberg, Victoria 3084, Australia. Email: fernando.calamante@florey.edu.au

Word count: 4777

Running title: DSC-MRI: deconvolution vs. summary parameters

Keywords: arterial input function, deconvolution, dynamic susceptibility contrast MRI, perfusion MRI, stroke. 


\begin{abstract}
Purpose: To investigate the performance of deconvolution-based parameters and summary parameters for quantifying dynamic-susceptibility contrast (DSC) MRI, with particular emphasis on their precision.
\end{abstract}

Methods: DSC-MRI quantification can be done using deconvolution with an arterial input function (AIF), or by using summary parameters measured directly from the concentration time-curves, e.g. time-to-peak. Despite early studies questioning the reliability of summary parameters, there is currently controversy regarding the benefits of deconvolution-based parameters in stroke imaging, with studies suggesting a similar infarct prediction using summary parameters. While deconvolution is supposed to eliminate any AIF-dependency, the regularisation step reintroduces some of this dependency. Numerical simulations were used to assess the contribution of noise and AIF variability to measurement precision.

Results: Deconvolution was found to considerably reduce the AIF-dependency, but a residual AIF bias remained on the calculated parameters. Summary parameters, in turn, show a lower sensitivity to noise. The residual AIF-dependency for deconvolution methods and the large AIF-sensitivity of summary parameters was greatly reduced when normalizing them relative to normal tissue.

Conclusions: Consistent with recent studies suggesting high performance of summary parameters in infarct prediction, results from this study suggest that DSC-MRI quantification using properly normalized summary parameters may have advantages in terms of lower noise and AIF-sensitivity as compared to commonly used deconvolution methods.

KEYOWRDS: arterial input function, deconvolution, dynamic susceptibility contrast MRI, perfusion MRI, stroke. 


\section{INTRODUCTION}

Dynamic-susceptibility contrast (DSC) MRI is a perfusion imaging technique frequently used in the workup of acute stroke patients (1). For quantification of cerebral blood flow (CBF), mean transit time (MTT), and the time-to-maximum of the tissue residue function (Tmax), the measurement of an arterial input function (AIF) and a deconvolution step are required (2). This is in contrast to socalled summary parameters derived from the tissue concentration time-course, such as its time-topeak (TTP) and first-moment (FM), which are calculated without deconvolution. However, early studies have questioned the reliability of these summary parameters based on their inherent dependence on multiple haemodynamic effects (e.g. bolus delay, dispersion, MTT) and on the patient-specific AIF $(3,4)$.

AIF-based processing no longer poses any significant computational burden, but it does require some sophistication, either by the user or by an automated software to identify the AIF. The AIF is highly susceptible to patient motion and multiple MR signal artefacts which may change the shape of the AIF and ultimately introduce error in the entire calculated map (5).

Interestingly, in the field of acute stroke imaging, there is currently some controversy regarding the benefits of the use of deconvolution-based parameters relative to quantification using summary parameters, with some studies suggesting that infarct prediction based on summary parameters is as good as (and, in some instances, even better than) the predictions from deconvolution-based parameters $(6,7)$. A possible reason for the reduced relative performance of deconvolution-based methods might be their higher signal-to-noise ratio (SNR) sensitivity compared to that of summary parameters. In fact, deconvolution is known for its very high noise-sensitivity, requiring some form of regularisation to stabilise the solution (8), which in turn often leads to quantification errors (e.g. larger overestimation of shorter MTT values)(9-11). Furthermore, while the commonly used Singular Value Decomposition (SVD) deconvolution based methods (9) are supposed to eliminate, in theory, any dependency on the shape of the AIF, the SVD technique is highly dependent on the 
AIF characteristics and the calculated maps are likely to maintain some degree of unwanted AIF dependency. However, this effect has not been investigated in detail yet.

In this study, the reproducibility of both deconvolution-based parameters and summary parameters across realistic noise and AIF shapes is investigated. While several previous studies have focussed on the accuracy of DSC-MRI quantification given one particular AIF shape (e.g. references (914)), less emphasis has been given to its precision (reproducibility) in a real world scenario where the AIF shape is highly variable. For a given physiological condition, the precision can be influenced by two factors: first, the effect of noise and, second, the AIF-sensitivity (i.e. the dependency on the specific shape of the AIF that remains despite the deconvolution). Therefore, there is a need to compare deconvolution-based parameters to summary parameters, in order to determine the limitations of each approach, and how they are influenced by each of these sources of variance. To this end, we first used patient data to characterise the range of AIFs for a typical acute stroke study. Numerical simulations were then carried out to synthesise dynamic DSC-MRI data with known ground-truth perfusion values, but using a large number of AIFs, each yielding a simulated dynamic acquisition of tissue with the same underlying perfusion values, but each based on a different AIF. An ideal deconvolution technique should always recover the ground truth values, independently of the AIF. We used established models for the tissue residue function, and a realistic range of tissue and vascular delay parameters. The simulated concentration time-curves were subsequently analysed using two popular SVD-based deconvolution algorithms $(9,10)$, as well as using summary parameters.

\section{METHODS}

\section{In vivo AIFs from patient data}

The DSC-MRI data included in this study were from acute stroke patients as part of the multi-site DEFUSE2 trial (15). The DEFUSE2 trial study protocol and patient-consent procedures were approved by Stanford's IRB following standard guidelines and regulations. Details of the imaging protocols have been reported previously (15). In brief, patients were imaged on a $1.5 \mathrm{~T}$ MRI 
scanner; using a gradient-echo PWI to follow a bolus of a gadolinium-based contrast-agent. Relevant parameters (15): $\mathrm{TE} / \mathrm{TR}=40 / 1800 \mathrm{~ms}, 14$ slices $(5 \mathrm{~mm}$ thickness), $128 \times 128$ matrix, $1.875 \mathrm{~mm}$ in-plane resolution, $60^{\circ}$ flip-angle. To ensure good data quality, datasets were excluded if they had substantial motion artefacts. Data from 23 patients were therefore included in this study (males/females $=11 / 12,39-86$ years, median 76 years; median time from symptoms onset $=4.8$ hours).

\section{Numerical simulations}

In order to investigate the AIF sensitivity of DSC-MRI quantification, a typical range of AIF curves that could be expected in clinical studies needs first to be determined. To this end, the AIF was measured from each in vivo dataset (to generate 23 AIFs) based on a semi-automatic searching algorithm (16). Each of these curves was then fitted to a gamma-variate function model (17): $\operatorname{AIF}(t) \sim t^{r} \times \exp (-t / b)$. (Note: in order to compare the shape of the curves from different patients, each fitted AIF was normalised to unit area, and the delay time set to 0). For an easier interpretation of the AIF distribution, the set of $(r, b)$ parameters were converted to $\mathrm{TTP}_{\mathrm{AIF}}$ and Maximum-PeakConcentration values, $\mathrm{MPC}_{\mathrm{AIF}}(4,18)$. From these set of values, an AIF distribution (Multivariate Gaussian) was determined (see Fig. 1a), resulting in a mean $\mathrm{TTP}_{\mathrm{AIF}}=6.58 \mathrm{~s}$ and mean $\mathrm{MPC}_{\mathrm{AIF}}=0.113$ (corresponding to $r_{\text {mean }}=3.66$ and $b_{\text {mean }}=1.80$ ), and an ellipsoid corresponding to its $95 \%$ percentile range, with covariance matrix:

$\Sigma=\left[\begin{array}{cc}0.240 & -0.349 \\ -0.349 & 0.933\end{array}\right]$

This percentile range was evenly sampled (44 points, based on a grid aligned with the ellipsoid) to generate a set of simulated AIFs from their corresponding $(r, b)$ values (Note: a further 15 points were evenly taken from the border of the ellipsoid, to characterise also the extremes of the interval). An illustrative sub-set of the corresponding simulated AIF curves is shown in Fig. 1b, which demonstrates that different patients can have very different AIF shapes, and that this variability must be taken into account in the simulations. 
Because the actual form of the in vivo residue function is still unclear, and it may vary between subjects, various published models were considered in this study:

a) mono-exponential decreasing function (9), corresponding to the most commonly used model: $\mathrm{R}(t, M T T)=\exp (-t / M T T)$;

b) gamma-distribution function (14):

$$
R(t, \lambda)=\int_{t}^{\infty} \frac{1}{\beta^{\lambda} \Gamma(\lambda)} t^{\lambda-1} e^{-\frac{t}{\beta}} d t
$$

with $\beta=\mathrm{MTT} / \lambda$ and $\Gamma(t)$ the Gamma function, which can resemble models between exponential and box functions, depending on $\lambda(14)$. In this study, $\lambda=5$ was chosen as an intermediate case between these two models; and

c) bi-exponential function (19):

$R\left(t, A, \tau_{1}, \tau_{2}\right)=A \times e^{-t \times \tau_{1}}+(1-A) \times e^{-t \times \tau_{2}}$

where $\mathrm{MTT}=A / \tau_{1}+(1-A) / \tau_{2}$.

Simulations were performed for MTT in the range 3-14 s (1 s increments), tissue arrival delays = $0-12 \mathrm{~s}$ ( $1 \mathrm{~s}$ increments), $\mathrm{CBV}=4 \%$, and $\mathrm{TR}=1.5 \mathrm{~s}$. This produced a total of $12 \times 13=156$ simulated haemodynamic situations. The resulting concentration time-curves were transformed to signal intensity curves, such that the tissue signal-drop (for normally perfused tissue, with MTT=4 s) was $40 \%$ for the case of the AIF corresponding to the mean of the distribution. Three noise levels were simulated $(\mathrm{SNR}=200,50,10)$, with 1000 noise realisations for each case (for a total of $\sim 500$ thousand curves for each AIF case). Figure 2 shows a schematic flow-chart of the simulation set up.

\section{Reference region}

In clinical and research practice, both summary and deconvolution-based perfusion parameters are often normalised to a contralateral reference region-of-interest (ROI) that is presumed normal. In order to capture this approach, we defined the simulated condition $\mathrm{CBV}=4 \%$, MTT $=4 \mathrm{~s}$, and 
delay $=0$ as 'normal', and used it to extract reference values for each parameter, as the mean value of the corresponding simulations.

\section{Deconvolution}

Two of the most popular deconvolution algorithms were assessed: SVD (9) and its delay-insensitive variant oSVD (10). Note: to explicitly distinguish the parameters calculated by these two methods, we use the prefix 'o' to indicate parameters calculated using oSVD (e.g. 'MTT' indicates the MTT calculated using SVD, and 'oMTT' that calculated using oSVD). In contrast to SVD (9), where the singular value expansion is truncated based on a uniform threshold for every voxel in every patient, an oscillation index in oSVD offers some adaptive level for the degree of regularisation (10), based on the data quality. However, given that the optimal degree of regularisation depends on SNR (10), different levels were considered for each SNR case:

- $\quad$ SVD threshold $=5 \%, 10 \%$ and $20 \%$, for SNR $=200,50$, and 10 respectively;

- $\quad$ oscillation index in $\mathrm{OSVD}=0.095,0.090$ and 0.085, for $\mathrm{SNR}=200,50$, and 10 respectively.

CBF and Tmax were then calculated from the maximum of the deconvolved residue function after Fourier interpolation $(20,21)(\times 4$ up-sampling); MTT was calculated from the CBV/CBF ratio.

\section{Summary parameters}

From the concentration time-curves, TTP and the normalised FM (i.e. ratio of the first to the zero-th moments) were calculated as summary parameters, as these are two of the most commonly used summary parameters in previous studies. As it was done for the deconvolution analysis, these parameters were calculated after Fourier interpolation $(\times 4$ up-sampling). Furthermore, to obtain a more robust estimate of TTP, this value was estimated from a quadratic fit to five (interpolated) points around the measured maximum concentration.

\section{Display of Results}

In order to visualize the influence of the AIF shape, the simulation results were reported either as 3D surface plots for the values across the AIF distribution (e.g. estimated Tmax values (in the $z$ - 
axis) as a function of the simulated AIF range (with the AIF shape parameters in the $x-y$ plane)), or as correlation plots (e.g. estimated MTT vs, true MTT). Unless otherwise indicated, all results correspond to the mono-exponential residue function case, given that it has been the more widely used model to date.

For a given physiological condition, measurement precision is determined by: i) noise, and ii) the variation in the shape of the AIF; there are therefore two sources of variability that must be quantified. This was done as follows: given that we have 1000 noise realisations and 59 simulated AIFs, there is a $59 \times 1000$ matrix for every combination of haemodynamic parameters and for each parameter map. For a given calculated parameter map $X$ (e.g. MTT), the elements of this matrix can be represented as $X_{i j}$, where $i=1, \ldots, 59$ and $j=1, \ldots, 1000$. When the mean over the 1000 noise realisations is computed (i.e. to calculate the average behaviour due to noise), one gets a $59 \times 1$ array (i.e. one result per AIF). The variation in this array is the variation due to the influence of the AIF, which was characterised by its standard deviation (STD) value, i.e.:

$\operatorname{STD}_{A I F}=\operatorname{STD} D_{\{i\}}\left[\operatorname{Mean}_{\{j\}}\left(X_{i j}\right)\right]$

where $\operatorname{Mean}_{\{j\}}$ indicates the mean over the $j$ dimension, and $S T D_{\{i\}}$ indicates the STD over the $i$ dimension.

Alternatively, if one computes the STD over the 1000 noise realisations (i.e. the variation due to noise), resulting in a $1 \times 59$ array, then the mean in this array will reflect the average variation due to noise, $\mathrm{STD}_{\text {noise, }}$ which was characterised by its mean value over the AIF range, i.e.:

$S T D_{\text {noise }}=\operatorname{Mean}_{\{i\}}\left[\operatorname{STD}_{\{j\}}\left(X_{i j}\right)\right]$

To visualise these two sources of variability, they were displayed as upper and lower error bars in the correlation plots:

- upper error-bar: standard deviation due to the noise;

- lower error-bar: standard deviation due to the AIF. 
The results were analysed both for absolute quantification, as well as for relative values (as difference, relative to the value from the reference ROI -see above).

\section{RESULTS}

$3 D$ surface plots

Figure 3 shows example surface plots for oMTT, oTmax and FM, as illustrative examples of the dependency of a given DSC-MRI parameter on the AIF distribution. All graphs on the left column (with absolute quantification) show a slope on the surface, meaning there is some AIF dependency (even for deconvolution-based parameters). As expected, the slope is much larger for summary parameters than for deconvolution-based parameters. The corresponding $95 \%$ percentile ranges were 1.3, 1.0, and $6.5 \mathrm{sec}$ in size, for oMTT, oTmax and FM, respectively. When the same results are calculated as relative values (right column), the slope of the surface is considerably reduced in every case, indicating that the AIF dependency is greatly reduced. This is particularly the case for summary parameters, where the very large slope is reduced considerably. In particular, the $95 \%$ percentile ranges were reduced to $0.2,0.4$, and 0.1 sec in size, for oMTT, oTmax and FM, respectively.

\section{Correlations plots}

Figure 4a shows the correlation plots for absolute MTT quantification, together with the errors due to noise and AIF (upper and lower error bars). Consistent with previous studies (9-11), both deconvolution algorithms overestimate very short MTT values. This figure also shows that the $S T D_{n o i s e}$ is larger for oSVD than for SVD. Furthermore, Fig. 4 shows that $S T D_{\text {noise }}$ increases for both SVD and oSVD with increasing MTT values. When considering the AIF dependency, oSVD performs better, with a lower $S T D_{A I F}$; this is as expected, because the regularisation method of oSVD is more adaptive (by the use of an oscillation-index), so it can vary depending on the AIF shape. Overall, the error of oSVD is mainly dominated by $S T D_{n o i s e}$, as the $S T D_{A I F}$ is very small; this is not the case for SVD. 
Figure $4 \mathrm{~b}$ shows the corresponding MTT results for relative quantitation, showing that oSVD gives more accurate results than SVD. Furthermore, $S T D_{A I F}$ is reduced for both SVD and oSVD. The oSVD result is still dominated by $S T D_{\text {noise }}$ and remains with a better performance than SVD.

Figure 5a shows the equivalent results for absolute Tmax quantification as a function of bolus delay - NB. Delay was chosen for the $\mathrm{x}$-axis in this case because Tmax is primarily a measure of delay (21). Consistent with previous studies (21), this figure shows that bolus delay has a simple additive effect on Tmax, and Tmax therefore provides a good estimate of delay, albeit by a fixed offset. In particular, a linear fit to the results indicate that this offset is $1.7 \mathrm{~s}$. Figure $5 \mathrm{a}$ also shows that $S T D_{\text {noise }}$ for Tmax is smaller for SVD than oSVD, consistent with earlier findings and the higher noise-sensitivity of oSVD. Moreover, as it was the case for MTT, $S T D_{A I F}$ is smaller for oSVD than for SVD. However, the actual $S T D_{A I F}$ and $S T D_{\text {noise }}$ values for Tmax were smaller than for MTT. Figure $5 \mathrm{~b}$ shows the corresponding results for Tmax for relative quantitation. As with MTT, the $S T D_{A I F}$ for oSVD reduces considerably and has a better performance than SVD.

Figure 6a shows the equivalent results for absolute quantification of the summary parameter First Moment, when plotted as a function of the simulated MTT. As expected, FM considerably overestimates the MTT value, because it also includes a contribution from the first moment of the AIF. This figure also shows that $S T D_{\text {noise }}$ is very small, much smaller than the values found for the deconvolution-based parameters; however, $S T D_{A I F}$ is very large. The FM results are, therefore, completely dominated by $S T D_{A I F}$. Figure $6 \mathrm{~b}$ shows the relative First Moment case, which shows a considerable decrease in $S T D_{A I F}$, as it was the case with Tmax and MTT, making both sources of variability (i.e. noise and AIF) very small.

Supporting Fig. S1a shows the equivalent results for First Moment displayed over different delays. As in Fig. 6a, $S T D_{A I F}$ dominates the result and is considerably larger than $S T D_{\text {noise }}$ In Supporting Fig. S1b, the corresponding results for relative FM are displayed; the same decrease in $S T D_{A I F}$ is seen, as it was the case for absolute quantification (Fig. 6b). 
Consistent with the FM results, the other summary parameter tested, TTP, was also found to severely overestimate MTT; similarly, $S T D_{A I F}$ was the dominating contribution to precision. $S T D_{n o i s e}$ and $S T D_{A I F}$ were found to increase with larger MTT; this increase was also seen for relative TTP quantification. Although the $S T D_{A I F}$ is reduced in relative numbers, it still increases with larger MTT. The results for TTP as a function of MTT can be found in the Supporting Fig. S2. When quantifying absolute TTP as a function of delay, the $S T D_{A I F}$ was found to dominate the results; this was reduced when relative TTP was considered. The $S T D_{\text {noise }}$ of TTP remained approximately the same over the whole delay range. For all cases, the STD values were larger for TTP than for FM. The results for TTP as a function of delay can be found in the Supporting Fig. S3.

\section{Effect of Residue models and SNR}

The results for $S T D_{A I F}$ and $S T D_{\text {noise }}$ were found to be qualitatively similar regardless of the specific residue model used in the simulations. In other words, the $S T D_{A I F}$ was found to be higher for SVD than oSVD, and $S T D_{\text {noise }}$ was higher for oSVD than for SVD, regardless of the residue model. As expected, however, the actual quantitative values depend of the residue model choice. Results for the various residue models can be found in the Supporting Fig. S4.

As expected, $S T D_{\text {noise }}$ was found to increase with decreasing SNR. However, the effect on $S T D_{A I F}$ was not as simple: while increasing noise had little effect on the AIF variability of Tmax, it reduced the variability for MTT (Note: Results for the various residue models can be found in the Supporting Fig. S5). Therefore, the qualitative results and conclusions from Figs. 4-6 and Supporting Figs. S1-S3 should be valid regardless of the particular simulated condition, although the actual quantitative value might change.

\section{DISCUSSION}

This study has a number of new important findings for DSC-MRI. These are described in turn below. 


\section{$3 D$ surface plots}

As could be expected, quantifying DSC-MRI parameters in relative units (relative to contralateral values) reduces the dependency on the AIF shape. In particular, this approach is essential when using summary parameters, given their very large AIF sensitivity. The results from Fig. 3 clearly showed that, despite this very large sensitivity to the specific shape of the AIF for TTP and FM, the sensitivity is greatly reduced (and, for FM, effectively eliminated) when referencing to contralateral values.

Interestingly, despite the use of deconvolution, the MTT parameter calculated from SVD showed a large AIF dependency (e.g. see also Fig. 4), likely related to the fixed threshold used for the SVD truncation (9), which is not effective in adapting the degree of regularisation for different AIF shapes. Our results suggest that MTT, when calculated from SVD, should not be used as an absolute parameter due to its imprecise nature.

Despite its limitations, the more adaptive regularisation in OSVD was found to lead to mild AIF sensitivity when quantifying in absolute units, suggesting that the contribution to precision due to the AIF shape probably can be neglected, even when quantifying in absolute units.

\section{Calculated MTT vs. simulated MTT}

As can be seen in Fig. 4, the oSVD method provides more accurate measurements than SVD, regardless whether MTT is quantified in absolute or relative units. The contributions to precision from noise and AIF sensitivity were, however, different: while $S T D_{\text {noise }}$ is higher for oSVD than for $\mathrm{SVD}$, this is the opposite for $S T D_{A I F}$, where $S T D_{A I F}$ in oSVD is greatly reduced to, in practice, negligible values. These results are likely due to the fact that the regularization method of oSVD uses an Oscillation Index (10), which provides more flexibility to allow for different thresholds to be used for different AIFs; this is in contrast to the fixed threshold for SVD, which fixes the level of regularisation regardless of the AIF shape. On the other hand, the more adaptive nature of the regularisation approach in oSVD does come at the expense of increased noise sensitivity, which is 
the reason for the associated larger $S T D_{\text {noise }}$ values. Overall, the precision of oSVD measurements (both in absolute and relative units) is, therefore, mainly dominated by $S T D_{\text {noise, }}$ as the $S T D_{A I F}$ is very small; this is not the case for SVD, where the two sources of variability are comparable.

\section{Calculated Tmax vs delay}

The accuracy of Tmax as a function of delay (Fig. 5a) was very similar, regardless of the deconvolution method used: consistent with previous studies (21), both SVD and oSVD show a linear Tmax increase with delay, with an offset of $1.7 \mathrm{sec}(\sim 1 \times \mathrm{TR})$, which is then eliminated when quantifying in relative units. As it was the case for MTT, the $S T D_{\text {noise }}$ is higher in oSVD, than SVD and $S T D_{A I F}$ is lower in oSVD than SVD. As expected, the $S T D_{\text {noise }}$ and $S T D_{A I F}$ where not dependent on delay for oSVD, consistent with the known delay-insensitivity of this deconvolution method (10)

\section{First Moment}

Consistent with previous studies $(3,4,23)$, FM provided a very inaccurate estimate of MTT, vastly overestimating this parameter. This is because, the first moment of the concentration time-curve $\left(\mathrm{FM}_{\mathrm{C}}\right)$ not only has a contribution from the first moment of the residue function $\left(\mathrm{FM}_{\mathrm{R}}\right)$, but also a contribution from the first moment of the AIF $\left(\mathrm{FM}_{\mathrm{AIF}}\right)$ :

$F M_{C}=F M_{R}+F M_{A I F}$

In contrast to deconvolution-based parameters, the contribution to FM precision from noise is very small, as can be seen in the $S T D_{\text {noise }}$ in Fig. 6 and Supporting Fig. S1. This is expected, because FM does not require a deconvolution step. In contrast, the AIF variation is very large and cannot be neglected: its contribution fully dominates the FM precision when quantified in absolute numbers, given that the concentration time-curve is highly dependent on the shape of the AIF.

When FM is expressed in relative values, the variation due to AIF reduces to negligible values. This can be explained based on Eq.[6]: by referencing to contralateral values, the $\mathrm{FM}_{\mathrm{AIF}}$ term disappears, 
making the results AIF-independent. However, it should be noted that the results will vary depending on the shape of the residue function, due to the $\mathrm{FM}_{\mathrm{R}}$ term.

\section{Time-to-Peak}

Consistent with previous studies (4), TTP was also found to provide an inaccurate estimate of MTT, severely overestimating its value. As it was the case for FM, the variability due to the AIF shape was larger than the contribution to precision due to noise. However, the $S T D_{\text {noise }}$ of TTP is considerable higher than that of FM, especially for tissues with long MTTs. This is due to the fact that, FM is calculated from a larger number of time points (cf. TTP calculated from a smaller number of time points around the peak concentration). This makes TTP estimation more sensitive to noise, particularly for wider concentration time-curves (i.e. from wider AIFs and/or long MTT values), where the estimated position of the maximum from broad peaks can be very noisedependent (Note: this is not the case for FM, given that the whole curve contributes to its estimate). This suggests that FM is preferable to TTP, when summary parameters are used for DSC-MRI quantification. However, it should be noted that FM will be more sensitive to motion, given that motion artefacts during the first passage of the bolus, but that occur after the maximum peakconcentration, will introduce errors in FM but not on TTP estimation. Once again, $S T D_{A I F}$ can be considerably reduced by quantifying relative TTP, making $S T D_{\text {noise }}$ the dominating factor.

\section{Residue Function model and SNR effects}

The results from the different models used to simulate the residue function show that the trends found, and by which conclusions in this study were drawn, are not restricted to the most commonly used model (the mono-exponential function). The qualitative similar results for the three very different residue function models tested provide support that our conclusions should be applicable to the in vivo case, where the actual shape of the residue function is not known. The same applies for the different SNR scenarios tested. 
We did not simulate situations where the residue function models in normal tissue and in abnormally perfused tissue were different. Such situations would bias FM and TTP maps by the shape of the residue function. In theory, deconvolution-based maps are not biased by the residue function model, but previous studies have shown that, in practice, there is a substantial difference in deconvolution performance with different underlying residue functions (10). As such, we do not expect this effect to change the conclusions of this study.

\section{Clinical implications}

A strong AIF dependency for a given parameter (e.g. absolute summary parameters, or even absolute MTT) suggests that the volume of the estimated perfusion abnormality does depend on the specific AIF shape; they are therefore not suited for diffusion-perfusion mismatch assessment (Note: that this is unrelated to the known delay-sensitivity of the SVD, given that the variability in question is only due to the AIF shape). In contrast, the simulations showed that some parameters had a much lower variability due to AIF (i.e. much smaller $S T D_{A I F}$ values), such it was the case for the relative First Moment and absolute Tmax, and would therefore provide a much more stable estimate of the mismatch volume.

\section{AIF distribution}

This study has characterised for the first time a realistic range of AIFs from clinical studies of adult subjects, which can be of great use in future numerical simulations of DSC-MRI. Studies using numerical simulations are playing a key role for the assessment and optimisation of analysis methods in DSC-MRI. The vast majority of these studies are based on the use of a single AIF curve, and therefore do not take into consideration the sensitivity to the shape of the input function.

Our simulations demonstrated that the simulated concentration-curves produced a wide variety of curves due to the different AIF shapes. As can be seen in Fig. 1b, the AIF shape can differ considerably from one another. Interestingly, while the AIF case most commonly used in previous numerical simulations (i.e., $r=3, b=1.5)(9)$ lies within the $95 \%$ percentile range distribution, it is in 
fact relatively distant from the mean of the distribution, and has a considerable different shape, more closely representing the shape of some of the narrowest AIFs in the distribution. It is therefore recommended that future studies using DSC-MRI simulations either simulate AIF from the whole distribution found in the current study or, at least, use the parameters corresponding to the mean of the distribution $\left(r_{\text {mean }}=3.66, b_{\text {mean }}=1.80\right)$ in order to simulate a more representative case.

\section{Limitations}

There are a number of limitations that should be mentioned. First, only two deconvolution algorithms were assessed; these were however the most commonly in used at present. The observed residual AIF sensitivity is likely related to the regularisation of the deconvolution, and points out to a limitation of the SVD methods tested, since they both have some degree of uniform regularisation (even for oSVD where a fixed oscillation index is used). It is expected that other deconvolution methods that have a more adaptive degree of regularisation (e.g. some iterative methods (11) and Bayesian-based methods $(14,24))$ may show a smaller AIF dependency. However, these methods tend to be much more computational demanding (and therefore with an increased processing time), which may not be feasible at present for some applications, such as in acute stroke. Secondly, time parameters (i.e. MTT and Tmax) were only considered among all deconvolution-based parameters; this is due to the fact that they are the most commonly used in stroke MRI studies. Thirdly, for patients with bilateral abnormalities it may be challenging to normalize to a region of normal tissue; AIF-based processing may be a preferred option in such cases. Fourthly, we have investigated the noise-characteristics and AIF-sensitivity of various types of DSC-MRI maps, as well as their sensitivity to haemodynamic delay and tissue MTT. It is not possible, however, to extrapolate these findings directly into their relative clinical performance.

\section{CONCLUSION}

The performance of different quantitative analysing methods for DSC-MRI data was evaluated. It was shown that, while deconvolution methods did considerably reduce the dependency on the AIF shape (compared with that of summary parameters), they had a residual AIF dependency, 
particularly so for MTT maps. Summary parameters, in turn, show a smaller variation due to noise. The residual AIF dependency for the SVD deconvolution methods and the large AIF sensitivity of summary parameters can be greatly reduced (and, in many cases, to negligible values), when quantification is carried out relative to the contralateral side. Our findings also suggest that new deconvolution algorithms should be evaluated in the context of multiple AIF. As shown in this study, AIF shape differences can have a significant contribution to the variability of the calculated perfusion values and an ideal deconvolution method should be minimally sensitive to these shape differences.

In summary, to get the best performance in terms of reduced AIF sensitivity; (i) MTT should be used as relative values; (ii) oMTT and oTmax are better as relative values, although their AIF sensitivity in absolute values is only minor and they could therefore have a role as absolute values; (iii) summary parameters FM and TTP must be used as relative values, if their AIF sensitivity is not to dominate the precision of the results.

Based on the current findings, and those from recent studies looking at lesion prediction $(6,7)$ quantification of DSC-MRI using summary parameters (i.e. without measuring an AIF or performing deconvolution) may provide a good alternative to AIF-based processing. Future studies are needed to clarify how maps of summary parameters (e.g. TTP and FM) compare to deconvolution-based maps (e.g. Tmax) for clinical decision making.

\section{ACKNOWLEDGEMENTS}

We are grateful to the National Health and Medical Research Council (NHMRC) of Australia, the Australian Research Council (ARC), and the Victorian Government's Operational Infrastructure Support Grant for their support.

\section{REFERENCES}

1. Wintermark M, Albers GW, Broderick JP, et al. Acute Stroke Imaging Research Roadmap II. Stroke 2013;44:2628-2639. 
2. Calamante F, Thomas DL, Pell GS, Wiersma J, Turner R. Measuring cerebral blood flow using magnetic resonance imaging techniques. J. Cereb. Blood Flow Metab. 1999;19:701-735.

3. Weisskoff RM, Chesler D, Boxerman JL, Rosen BR. Pitfalls in MR measurement of tissue blood flow with intravascular tracers: which mean transit time? Magn. Reson. Med. 1993;29:553-558.

4. Perthen JE, Calamante F, Gadian DG, Connelly A. Is quantification of bolus tracking MRI reliable without deconvolution? Magn. Reson. Med. 2002;47:61-67.

5. Calamante F. Arterial input function in perfusion MRI: a comprehensive review. Prog. Nucl. Magn. Reson. Spectrosc. 2013;74:1-32.

6. Christensen S, Mouridsen K, Wu O, Hjort N, Karstoft H, Thomalla G, Röther J, Fiehler J, Kucinski T, Østergaard L. Comparison of 10 perfusion MRI parameters in 97 sub-6-hour stroke patients using voxel-based receiver operating characteristics analysis. Stroke 2009;40:20552061.

7. Willats L, Connelly A, Christensen S, Donnan GA, Davis SM, Calamante F. The role of bolus delay and dispersion in predictor models for stroke. Stroke 2012;43:1025-1031.

8. Willats L, Calamante F. The 39 steps: evading error and deciphering the secrets for accurate dynamic susceptibility contrast MRI. NMR Biomed. 2013;26:913-931.

9. Ostergaard L, Weisskoff RM, Chesler DA, Gyldensted C, Rosen BR. High resolution measurement of cerebral blood flow using intravascular tracer bolus passages. Part I: Mathematical approach and statistical analysis. Magn. Reson. Med. 1996;36:715-725.

10. Wu O, Østergaard L, Weisskoff RM, Benner T, Rosen BR, Sorensen AG. Tracer arrival timinginsensitive technique for estimating flow in MR perfusion-weighted imaging using singular value decomposition with a block-circulant deconvolution matrix. Magn. Reson. Med. $2003 ; 50: 164-174$. 
11. Willats L, Connelly A, Calamante F. Minimising the effects of bolus dispersion in bolustracking MRI. NMR Biomed. 2008;21:1126-1137.

12. Willats L, Connelly A, Calamante F. Improved deconvolution of perfusion MRI data in the presence of bolus delay and dispersion. Magn. Reson. Med. 2006;56:146-156.

13. Calamante F, Gadian DG, Connelly A. Quantification of bolus-tracking MRI: Improved characterization of the tissue residue function using Tikhonov regularization. Magn. Reson. Med. 2003;50:1237-1247.

14. Mouridsen K, Friston K, Hjort N, Gyldensted L, Østergaard L, Kiebel S. Bayesian estimation of cerebral perfusion using a physiological model of microvasculature. NeuroImage 2006;33:570579.

15. Lansberg MG, Straka M, Kemp S, et al. MRI profile and response to endovascular reperfusion after stroke (DEFUSE 2): a prospective cohort study. Lancet Neurol. 2012;11:860-867.

16. Mouridsen K, Christensen S, Gyldensted L, Ostergaard L. Automatic selection of arterial input function using cluster analysis. Magn. Reson. Med. 2006;55:524-531.

17. Thompson HK Jr, Starmer CF, Whalen RE, Mcintosh HD. Indicator transit time considered as a gamma variate. Circ. Res. 1964;14:502-515.

18. Madsen MT. A simplified formulation of the gamma variate function. Phys. Med. Biol. 1992;37:1597-1600.

19. Mehndiratta A, Calamante F, MacIntosh BJ, Crane DE, Payne SJ, Chappell MA. Modeling the residue function in DSC-MRI simulations: analytical approximation to in vivo data. Magn. Reson. Med. 2014;72:1486-1491. 
20. Salluzzi M, Frayne R, Smith MR. Is correction necessary when clinically determining quantitative cerebral perfusion parameters from multi-slice dynamic susceptibility contrast MR studies? Phys. Med. Biol. 2006;51:407-424.

21. Calamante F, Christensen S, Desmond PM, Ostergaard L, Davis SM, Connelly A. The physiological significance of the time-to-maximum (Tmax) parameter in perfusion MRI. Stroke J. Cereb. Circ. 2010;41:1169-1174.

22. Olivot J-M, Mlynash M, Thijs VN, Kemp S, Lansberg MG, Wechsler L, Bammer R, Marks MP, Albers GW. Optimal Tmax threshold for predicting penumbral tissue in acute stroke. Stroke 2009;40:469-475.

23. Christensen S, Calamante F, Hjort N, Wu O, Blankholm AD, Desmond P, Davis S, Ostergaard L. Inferring origin of vascular supply from tracer arrival timing patterns using bolus tracking MRI. J. Magn. Reson. Imaging 2008;27:1371-1381.

24. Mehndiratta A, Macintosh BJ, Crane DE, Payne SJ, Chappell MA. A control point interpolation method for the non-parametric quantification of cerebral haemodynamics from dynamic susceptibility contrast MRI. NeuroImage 2012;64C:560-570.

\section{FIGURE LEGENDS}

Figure 1: (a) AIF distribution from in vivo data. Each blue cross corresponds to the AIF from one patient, and is characterised by its $\mathrm{TTP}_{\mathrm{AIF}}$ and $\mathrm{MPC}_{\mathrm{AIF}}$ values. The distribution was modelled as a multivariate Gaussian, with the mean indicated by the red circle and the $95 \%$ percentile range by the ellipsoid. The black rhombus indicates the location of the widely used AIF model $(r=3, b=1.5)$ (9), labelled 'Traditional' in the legend. (b) Example AIF curves simulated from samples in the distribution described in (a); each curve is labelled by its $\left[\mathrm{TTP}_{\mathrm{AIF}} ; \mathrm{MPC}_{\mathrm{AIF}}\right]$ values. The ticker blue line corresponds to mean of the AIF distribution. The dashed line represents the shape of the widely 
used AIF model. The figure illustrates the range of AIF shapes that can be found in vivo. It also shows that the widely used AIF model is very different from the shape of the mean of the distribution, and it more closely represents some of the narrowest AIFs in the distribution.

Figure 2: Schematic flow-chart of simulation set up. Top left: AIF cases (Note: although 5 cases are shown, 59 were considered for the simulations). Bottom left: residue function models. For each model, a range of MTT and CBV values were considered. Top right: tissue concentration time course; a range of delay values, SNR cases and noise realisations were considered. The tissue curves were analysed either using deconvolution (SVD and oSVD) or using summary parameters.

Figure 3: Example 3D surface plots. Left column (a,c,e) shows the surface plots (calculated in absolute units) for oMTT, oTmax and First Moment, respectively. The right column (b,d,f) show the corresponding plots when quantified in relative units. All plots are generated using $\mathrm{CBV}=4 \%$, $\mathrm{MTT}=10 \mathrm{~s}$, and delay $=0 \mathrm{~s}$. For all parameters, the length of the range of z-axis scale was kept constant (an 8-sec range fort all) to help visualisation of the reduced AIF-dependency for quantification using relative values.

Figure 4: Example correlation plots for MTT and oMTT as a function of true MTT. (a) Absolute quantification; (b) relative quantification. Black solid lines indicate the results using oSVD, and the grey lines the results using SVD. Data were simulated using the mono-exponential residue function, $\mathrm{CBV}=4 \%$, delay $=0 \mathrm{~s}$, and $\mathrm{SNR}=200$. The dashed line corresponds to the identity line. The upper/lower error-bars indicate the standard deviation due to the noise/AIF. For ease of visualisation of the error bars, the x-values for the results from SVD and oSVD were slightly shifted in opposite directions, to reduce their overlap.

Figure 5: Example correlation plots for Tmax and oTmax as a function of true delay. (a) Absolute quantification; (b) relative quantification. Black solid lines indicate the results using oSVD, and the grey lines the results using SVD. Data were simulated using the mono-exponential residue function, $\mathrm{CBV}=4 \%, \mathrm{MTT}=4 \mathrm{~s}$, and $\mathrm{SNR}=200$. The dashed line corresponds to the identity line. The upper/lower error-bars indicate the standard deviation due to the noise/AIF. For ease of Magnetic Resonąnce in Medicine 
visualisation of the error bars, the x-values for the results from SVD and oSVD were slightly shifted in opposite directions, to reduce their overlap.

Figure 6: Example correlation plots for first moment as a function of true MTT. (a) Absolute quantification; (b) relative quantification. Data were simulated using the mono-exponential residue function, $\mathrm{CBV}=4 \%$, delay $=0 \mathrm{~s}$, and $\mathrm{SNR}=200$. The upper/lower error-bars indicate the standard deviation due to the noise/AIF.

\section{SUPPORTING FIGURE LEGENDS}

Supporting Figure S1: Example correlation plots for first moment as a function of true delay. (a) Absolute quantification; (b) relative quantification. Data were simulated using the monoexponential residue function, $\mathrm{CBV}=4 \%, \mathrm{MTT}=4 \mathrm{~s}$, and $\mathrm{SNR}=200$. The upper/lower error-bars indicate the standard deviation due to the noise/AIF.

Supporting Figure S2: Example correlation plots for TTP as a function of true MTT. (a) Absolute quantification; (b) relative quantification. Data were simulated using the mono-exponential residue function, $\mathrm{CBV}=4 \%$, delay $=0 \mathrm{~s}$, and $\mathrm{SNR}=200$. The upper/lower error-bars indicate the standard deviation due to the noise/AIF.

Supporting Figure S3: Example correlation plots for TTP as a function of true delay. (a) Absolute quantification; (b) relative quantification. Data were simulated using the mono-exponential residue function, $\mathrm{CBV}=4 \%, \mathrm{MTT}=4 \mathrm{~s}$, and $\mathrm{SNR}=200$. The upper/lower error-bars indicate the standard deviation due to the noise/AIF.

Supporting Figure S4: Effect of residue function model on the results of the correlation plots. Example correlation plots for: (a) MTT and oMTT as a function of true MTT, and (b) Tmax and oTmax as a function of true delay. Solid colour lines indicate the results using SVD, and the dashed colour lines the results using oSVD, with the different colours labelling the various residue models (Model 1: mono-exponential, Model 2: Gamma-distribution function, Model 3: Bi-exponential). These results correspond to absolute quantification and simulations performed using the mono- 
exponential residue function, $\mathrm{CBV}=4 \%$, and $\mathrm{SNR}=200$. The dashed line corresponds to the identity line. The upper/lower error-bars indicate the standard deviation due to the noise/AIF. For ease of visualisation of the error bars, the x-values for the results from SVD and oSVD were slightly shifted in opposite directions, to reduce their overlap.

Supporting Figure S5: Effect of noise level on the results of the correlation plots. Example correlation plots for: (a) oMTT as a function of true MTT, and (b) oTmax as a function of true delay. The different colours indicate the various SNR levels. These results correspond to absolute quantification using oSVD, and simulations performed using the mono-exponential residue function, $\mathrm{CBV}=4 \%$, and $\mathrm{SNR}=200$. The dashed line corresponds to the identity line. The upper/lower error-bars indicate the standard deviation due to the noise/AIF. For ease of visualisation of the error bars, the x-values for the results from each SNR condition were slightly shifted relative to each other, to reduce their overlap. 

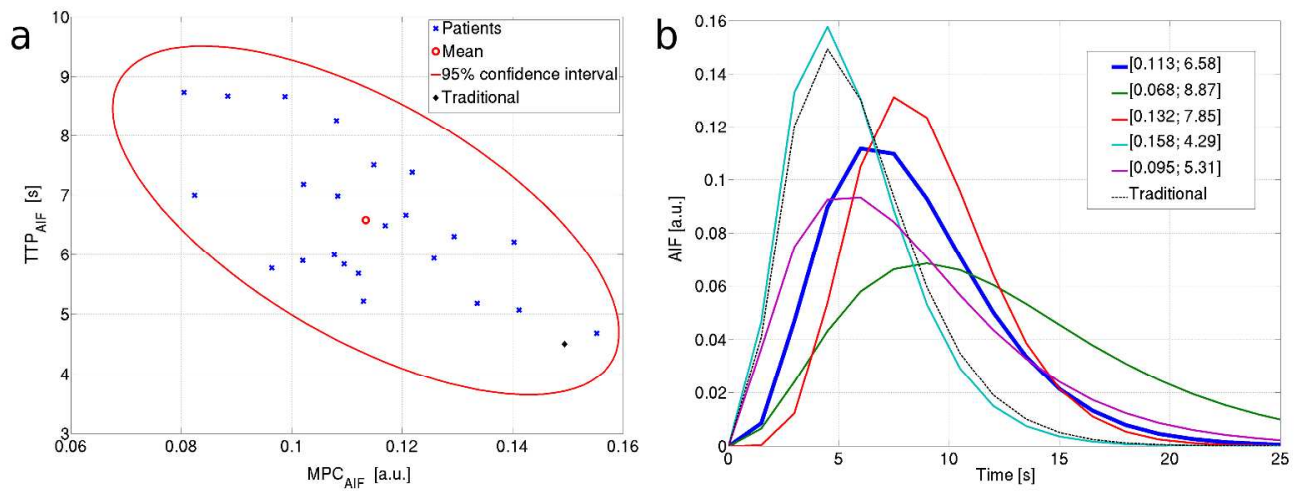

Figure 1: (a) AIF distribution from in vivo data. Each blue cross corresponds to the AIF from one patient, and is characterised by its TTPAIF and MPCAIF values. The distribution was modelled as a multivariate Gaussian, with the mean indicated by the red circle and the $95 \%$ percentile range by the ellipsoid. The black rhombus indicates the location of the widely used AIF model $(r=3, b=1.5)(9)$, labelled 'Traditional' in the legend. (b) Example AIF curves simulated from samples in the distribution described in (a); each curve is labelled by its [TTPAIF; MPCAIF] values. The ticker blue line corresponds to mean of the AIF distribution. The dashed line represents the shape of the widely used AIF model. The figure illustrates the range of AIF shapes that can be found in vivo. It also shows that the widely used AIF model is very different from the shape of the mean of the distribution, and it more closely represents some of the narrowest AIFs in the distribution.

$795 \times 293 \mathrm{~mm}$ (157 x 161 DPI) 
1

2

3

4

5

6

7

8

9

10

11

12

13

14

15

16

17

18

19

20

21

22

23

24

25

26

27

28

29

30

31

32

33

34

35

36

37

38

39

40

41

42

43

44

45

46

47

48

49

50

51

52

53

54

55

56

57

58

59

60
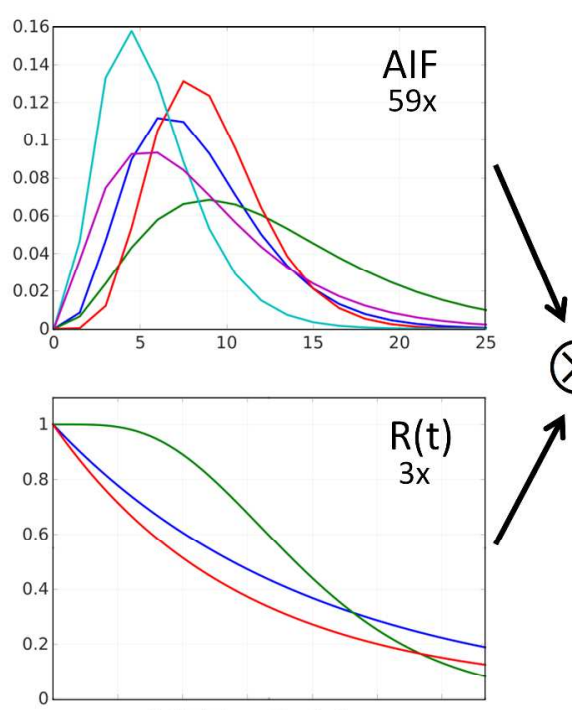

$\mathrm{MTT}=3-14 \mathrm{~s}$

CBV $=4 \%$

Figure 2: Schematic flow-chart of simulation set up. Top left: AIF cases (Note: although 5 cases are shown, 59 were considered for the simulations). Bottom left: residue function models. For each model, a range of MTT and CBV values were considered. Top right: tissue concentration time course; a range of delay values, SNR cases and noise realisations were considered. The tissue curves were analysed either using deconvolution (SVD and OSVD) or using summary parameters.

$$
1130 \times 700 \mathrm{~mm}(96 \times 96 \mathrm{DPI})
$$



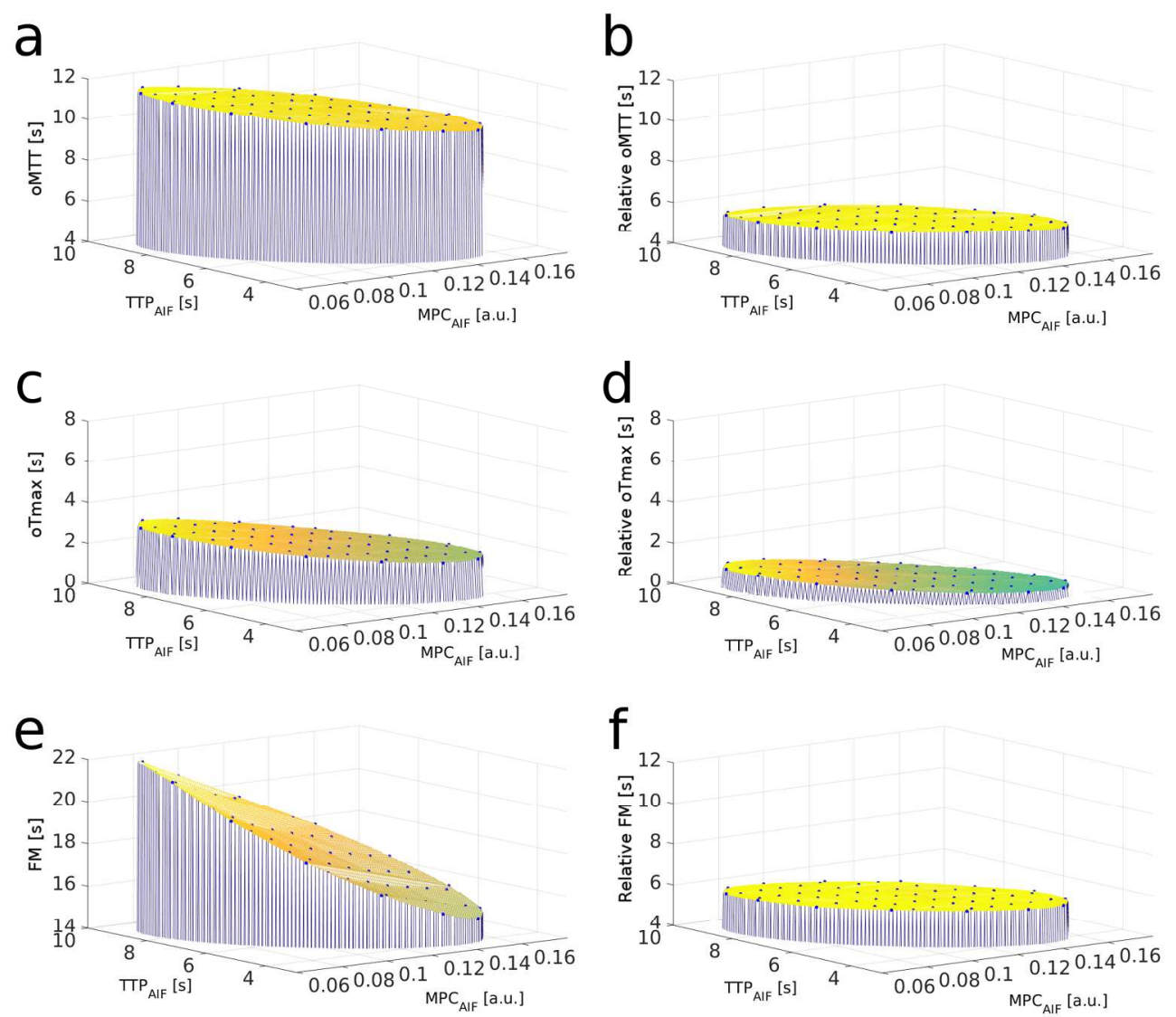

Figure 3: Example 3D surface plots. Left column $(a, c, e)$ shows the surface plots (calculated in absolute units) for oMTT, oTmax and First Moment, respectively. The right column $(b, d, f)$ show the corresponding plots when quantified in relative units. All plots are generated using $C B V=4 \%, M T T=10$ s, and delay $=0$ s. For all parameters, the length of the range of z-axis scale was kept constant (an 8-sec range fort all) to help visualisation of the reduced AIF-dependency for quantification using relative values.

$541 \times 474 \mathrm{~mm}(96 \times 96 \mathrm{DPI})$ 

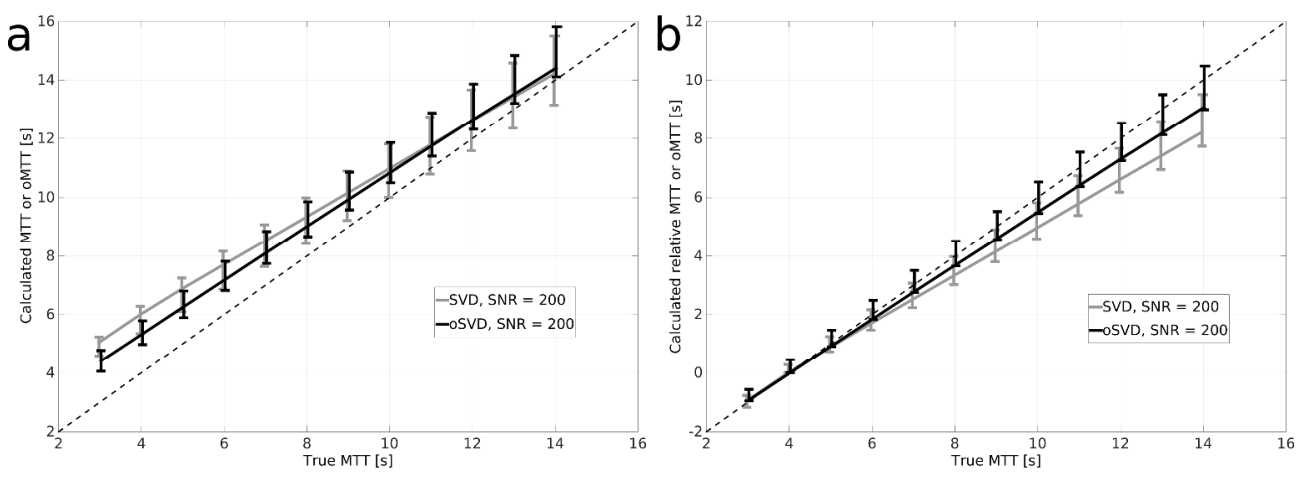

Figure 4: Example correlation plots for MTT and oMTT as a function of true MTT. (a) Absolute quantification; (b) relative quantification. Black solid lines indicate the results using OSVD, and the grey lines the results using SVD. Data were simulated using the mono-exponential residue function, $C B V=4 \%$, delay $=0 \mathrm{~s}$, and $S N R=200$. The dashed line corresponds to the identity line. The upper/lower error-bars indicate the standard deviation due to the noise/AIF. For ease of visualisation of the error bars, the $x$-values for the results from SVD and OSVD were slightly shifted in opposite directions, to reduce their overlap. $1275 \times 460 \mathrm{~mm}(96 \times 96 \mathrm{DPI})$ 

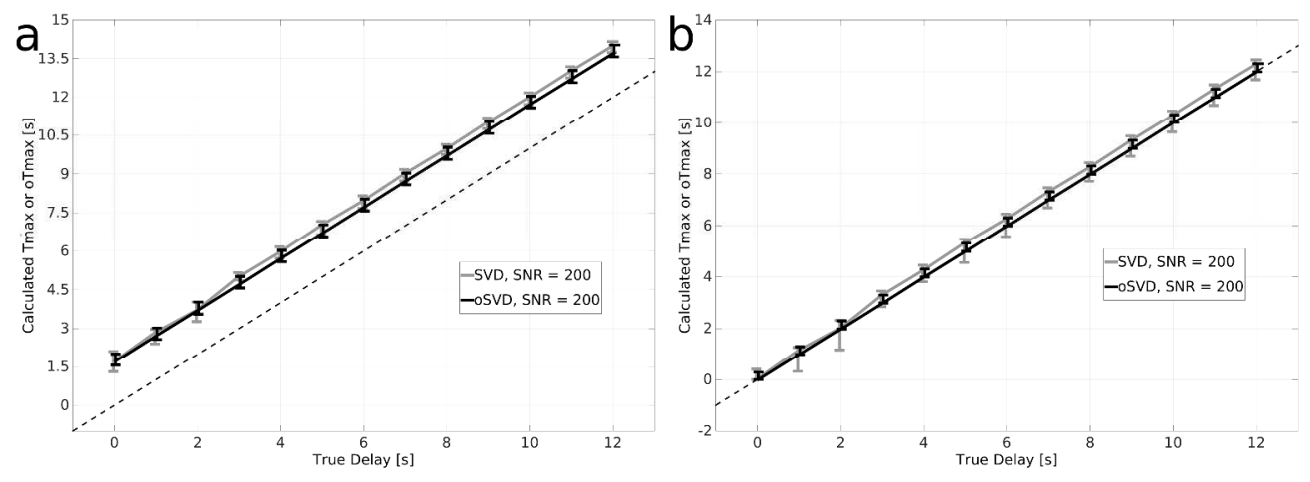

Figure 5: Example correlation plots for Tmax and oTmax as a function of true delay. (a) Absolute quantification; (b) relative quantification. Black solid lines indicate the results using OSVD, and the grey lines the results using SVD. Data were simulated using the mono-exponential residue function, $C B V=4 \%$, $M T T=4 s$, and SNR=200. The dashed line corresponds to the identity line. The upper/lower error-bars indicate the standard deviation due to the noise/AIF. For ease of visualisation of the error bars, the $x$-values for the results from SVD and OSVD were slightly shifted in opposite directions, to reduce their overlap. $1273 \times 464 \mathrm{~mm}(96 \times 96 \mathrm{DPI})$ 
1

2

3

4

5

6

7

8

9

10

11

12

13

14

15

16

17

18

19

20

21

22

23

24

25

26

27

28

29

30

31

32

33

34

35

36

37

38

39

40

41

42

43

44

45

46

47

48

49

50

51

52

53

54

55

56

57

58

59

60
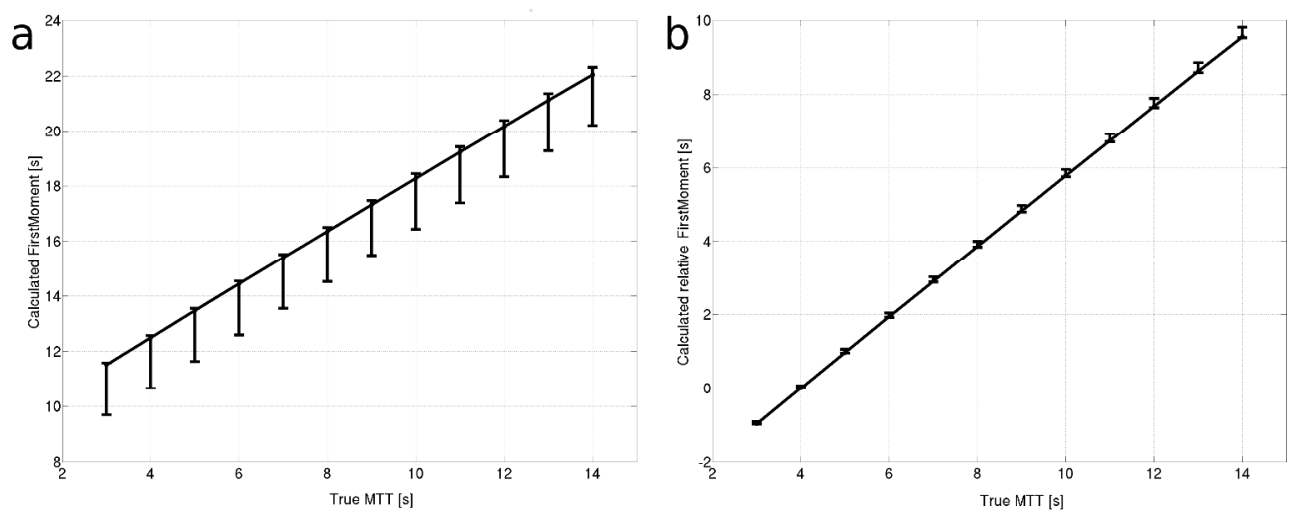

Figure 6: Example correlation plots for first moment as a function of true MTT. (a) Absolute quantification; (b) relative quantification. Data were simulated using the mono-exponential residue function, $\mathrm{CBV}=4 \%$, delay $=0 \mathrm{~s}$, and $S N R=200$. The upper/lower error-bars indicate the standard deviation due to the noise/AIF. $724 \times 277 \mathrm{~mm}(157 \times 161 \mathrm{DPI})$ 

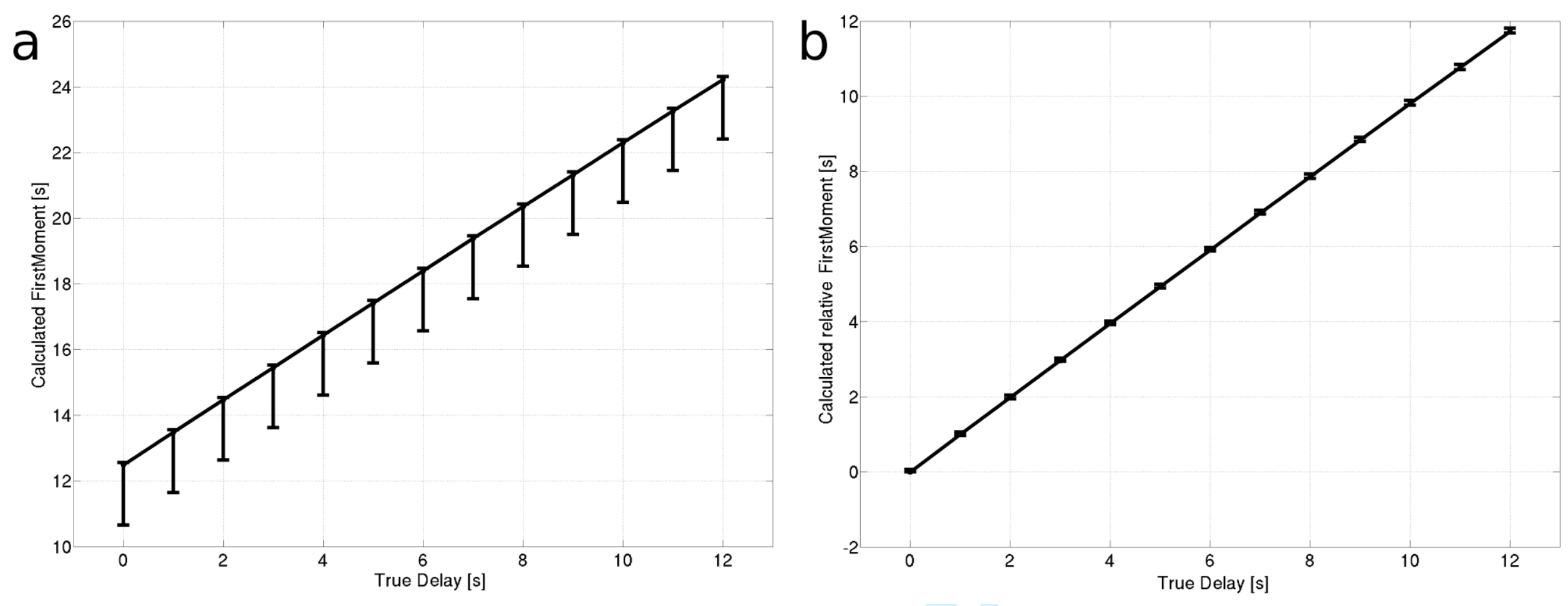

Supporting Figure S1: Example correlation plots for first moment as a function of true delay. (a) Absolute quantification; (b) relative quantification. Data were simulated using the mono-exponential residue function, $\mathrm{CBV}=4 \%$, MTT=4s, and $\mathrm{SNR}=200$. The upper/lower error-bars indicate the standard deviation due to the noise/AIF. 

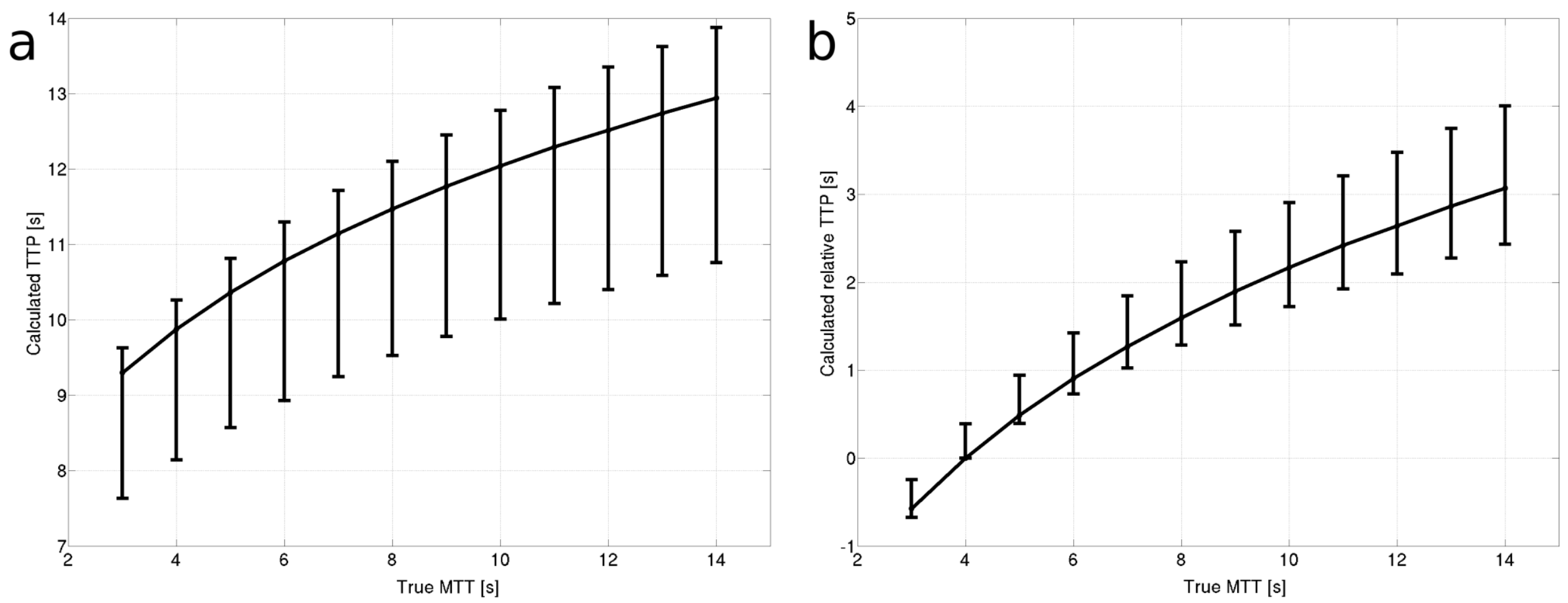

Supporting Figure S2: Example correlation plots for TTP as a function of true MTT. (a) Absolute quantification; (b) relative quantification. Data were simulated using the mono-exponential residue function, $\mathrm{CBV}=4 \%$, delay=0s, and $\mathrm{SNR}=200$. The upper/lower error-bars indicate the standard deviation due to the noise/AIF. 

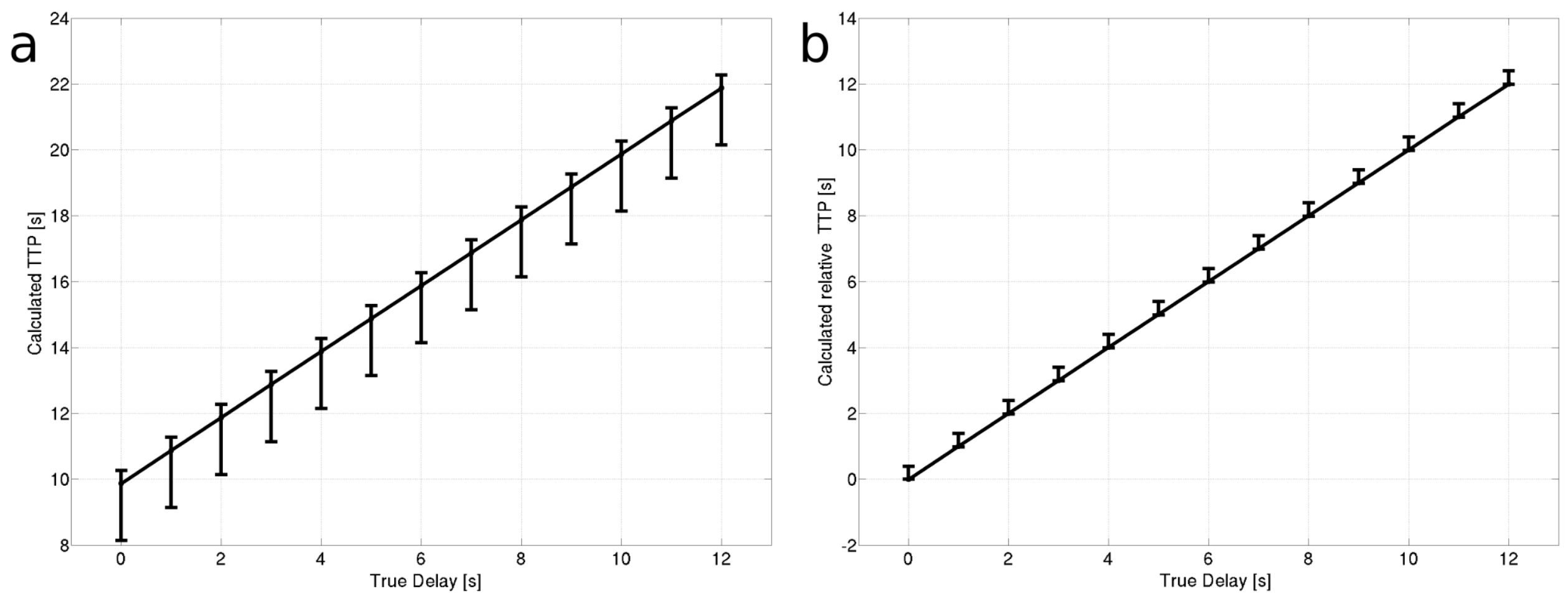

Supporting Figure S3: Example correlation plots for TTP as a function of true delay. (a) Absolute quantification; (b) relative quantification. Data were simulated using the mono-exponential residue function, $\mathrm{CBV}=4 \%, \mathrm{MTT}=4 \mathrm{~s}$, and $\mathrm{SNR}=200$. The upper/lower error-bars indicate the standard deviation due to the noise/AIF. 

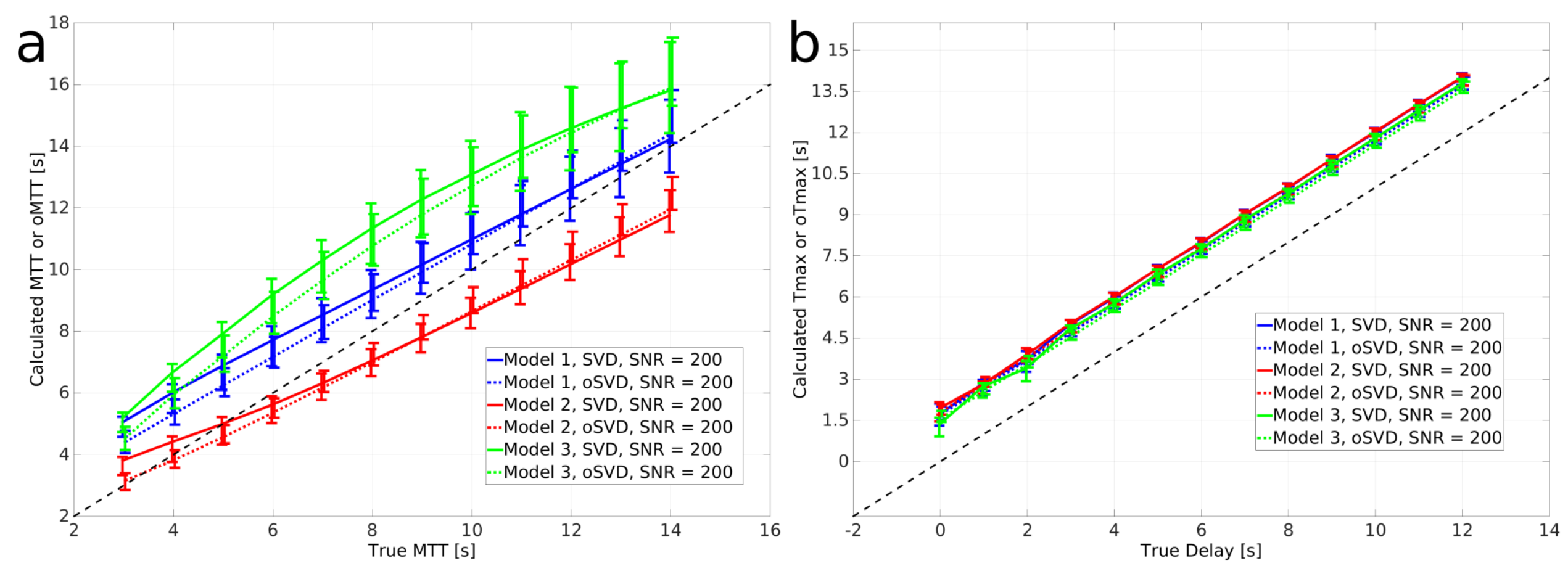

Supporting Figure S4: Effect of residue function model on the results of the correlation plots. Example correlation plots for: (a) MTT and oMTT as a function of true MTT, and (b) Tmax and oTmax as a function of true delay. Solid colour lines indicate the results using SVD, and the dashed colour lines the results using oSVD, with the different colours labelling the various residue models (Model 1: mono-exponential, Model 2: Gammadistribution function, Model 3: Bi-exponential). These results correspond to absolute quantification and simulations performed using the monoexponential residue function, $\mathrm{CBV}=4 \%$, and $\mathrm{SNR}=200$. The dashed line corresponds to the identity line. The upper/lower error-bars indicate the standard deviation due to the noise/AIF. For ease of visualisation of the error bars, the x-values for the results from SVD and oSVD were slightly shifted in opposite directions, to reduce their overlap. 

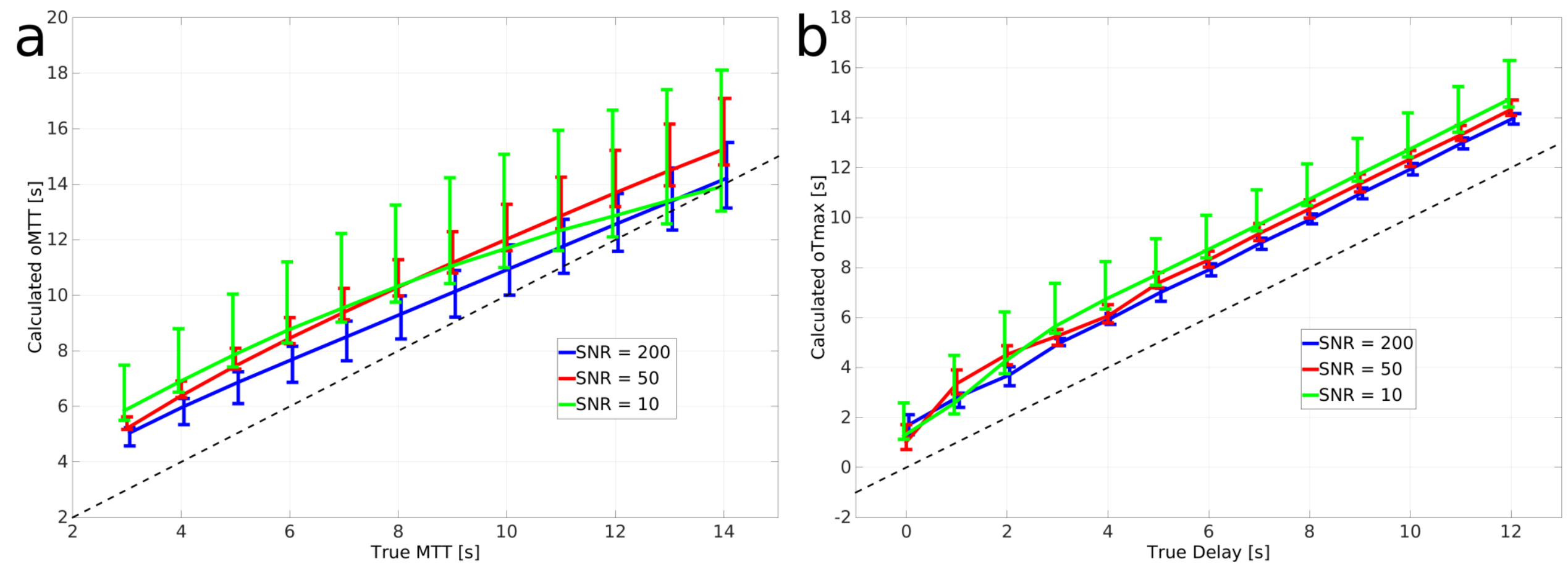

Supporting Figure S5: Effect of noise level on the results of the correlation plots. Example correlation plots for: (a) oMTT as a function of true MTT, and (b) oTmax as a function of true delay. The different colours indicate the various SNR levels. These results correspond to absolute quantification using oSVD, and simulations performed using the mono-exponential residue function, $\mathrm{CBV}=4 \%$, and $\mathrm{SNR}=200$. The dashed line corresponds to the identity line. The upper/lower error-bars indicate the standard deviation due to the noise/AIF. For ease of visualisation of the error bars, the x-values for the results from each SNR condition were slightly shifted relative to each other, to reduce their overlap. 


\section{University Library}

\section{- M M N E R VA A gateway to Melbourne's research publications}

Minerva Access is the Institutional Repository of The University of Melbourne

Author/s:

Meijs, M;Christensen, S;Lansberg, MG;Albers, GW;Calamante, F

Title:

Analysis of Perfusion MRI in Stroke: To Deconvolve, or not to Deconvolve

Date:

2016-10-01

Citation:

Meijs, M., Christensen, S., Lansberg, M. G., Albers, G. W. \& Calamante, F. (2016). Analysis of Perfusion MRI in Stroke: To Deconvolve, or not to Deconvolve. MAGNETIC RESONANCE IN MEDICINE, 76 (4), pp.1282-1290. https://doi.org/10.1002/mrm.26024.

Persistent Link:

http://hdl.handle.net/11343/91987 